\title{
Neoraimondia arequipensis subsp. roseiflora (Werdermann \& Backeberg) Ostolaza (Cactaceae): EJE DE LAS INTERACCIONES EN ECOSISTEMAS ÁRIDOS
}

\section{Neoraimondia arequipensis subsp. roseiflora (Werdermann \& Backeberg) Ostolaza(Cactaceae): AXIS OF THE INTERACTIONS IN ARID ECOSYSTEMS}

\author{
Aldo Ceroni Stuva ${ }^{1}$, Viviana Castro Cepero ${ }^{1}$, Vanessa Teixeira Roth ${ }^{3}$ e Inés Redolfi Pivatto ${ }^{2}$
}

\section{Resumen}

Se realizó una revisión de artículos del cactus endémico para el Perú Neoraimondia arequipensis subsp. roseiflora (Werdermann \& Backeberg) Ostolaza. Estudios realizados demuestran una relación estrecha del cactus con el ecosistema, a través de una serie de interacciones tanto con el suelo, como planta nodriza, en la polinización, la dispersión y con la entomofauna, reforzando la hipótesis que este cactus tiene un rol muy importante en ecosistemas áridos y se constituye en el eje central en la cadena trófica de una serie de organismos. Se plantea también futuros estudios a realizarse a fin de comprender mejor el rol que juega $N$. arequipensis subsp. roseiflora como eje de las interacciones en ecosistemas áridos en el Perú y en la dinámica de las poblaciones y la estructura de la comunidad del desierto.

Palabras clave: ecosistemas áridos, entomofauna, fenología, interacciones, nodricismo

\begin{abstract}
A literature review on Neoraimondia arequipensis subsp. roseiflora (Werdermann \& Backeberg) Ostolaza (1998), an endemic cactus in Peru, was performed. Previous researches show that there is a strong relation between the cactus and the ecosystem. This relation is manifested through interactions between this cactus and nurse plants, soil, pollination, seed dispersal, and surrounding enthomofauna, which reinforces the hypothesis that this cactus has a very important role in arid ecosystems and that it is the central axis in the trophic chain of a group of organisms. Finally, we outline future studies that could be carried out in order to understand the role played by this cactus as an axis of the interactions in arid ecosystems in Peru, as well as population's dynamics and community structure in the desert.
\end{abstract}

Key words: arid ecosystems, enthomofauna, fenology, interactions, nodricism

\section{Introducción}

Neoraimondia arequipensis subsp. roseiflora (Werdermann \& Backeberg) Ostolaza (1998) es un cactus endémico en el Perú y se encuentra ampliamente distribuido a través de los Andes, en los Departamentos de Ancash, Lima e Ica, siendo el Departamento de Lima en donde su presencia es verificada en casi todos los valles con un alto número de individuos. Esta especie puede llegar a tener un gran porte, con una estructura columnar robusta y ramificada desde la base. Estudios realizados en los últimos años han permitido demostrar una relación estrecha entre los componentes del suelo como el porcentaje de materia orgánica y disponibilidad de fósforo y potasio, y la densidad de este cactus. Por otro lado, se sabe que el crecimiento bajo plantas nodrizas afecta también la distribución de los cactus, haciendo que estos presenten una distribución agregada, lo que puede representar una interacción relevante entre el cactus y su nodriza en las comunidades de zonas áridas. Para el caso de $N$. arequipensis subsp. roseiflora también se ha encontrado una distribución agregada con otros cactus indicando una posible relación de nodricismo entre este y los otros cactus de menor porte creciendo bajo su sombra. Asimismo, la fenología del cactus presenta una interacción con una serie de organismos en torno a él como con la entomofauna, todo lo cual reforzaría la hipótesis que este cactus tiene un rol muy importante en ecosistemas áridos y se constituye en el eje central en la cadena trófica de una serie de organismos. El presente artículo tiene por finalidad hacer una revisión de los trabajos realizados hasta el momento con $N$. arequipensis subsp. roseiflora, así como también plantear los futuros estudios a realizarse a fin de comprender mejor el rol que juega este importante cactus como eje de las interacciones en ecosistemas áridos en el Perú y en la dinámica de las poblaciones y la estructura de la comunidad del desierto.

\section{Taxonomía}

La especie Neoraimondia arequipensis subsp. roseiflora, perteneciente a familia Cactaceae, sub familia Cactoideae, tribu Browningieae, fue descrita originalmente por Meyen en 1833 como Cereus arequipensis y más tarde redescrita por Schumann en 1903 como Pilocereus macrostibas (Anderson, 2001). En 1920, Britton \& Rose (1919-1922) publican el 
género Neoraimondia en honor al naturalista italiano, estudioso de la flora y geografía del Perú, Antonio Raimondi (1825-1890), basándose en la descripción hecha por Schumann; la especie es tratada como Neoraimondia macrostibas (Schumann) Britton \& Rose, caracterizada por sus abultadas areolas, espinas de hasta $25 \mathrm{~cm}$ y flores blancas. Según Ostolaza et al. (1985) y Anderson (2001), Backeberg demostró en base a comparaciones de material vegetal y en las descripciones originales, que las especies descritas como Cereus arequipensis, Pilocereus macrostibas y Neoraimondia macrostibas, corresponden a la misma planta. Backeberg la publicó en 1936 como Neoraimondia arequipensis (Meyen) Backeberg.

La especie presenta dos subespecies que se diferencian por el color de la flor, tamaño y el número de costillas. Una de estas subespecies ya había sido publicada previamente como Neoraimondia roseiflora (Werdermann \& Backeberg) Backeberg en 1932. Esta subespecie es tratada por Ostolaza (1996) bajo el nombre de Neoraimondia arequipensis var. roseiflora (Backeberg) Ritter, para tipificar la población cuya variación se encuentra en el color rosado de las flores. Sin embargo, Hunt (1999) la reporta como Neoraimondia arequipensis (Meyen) Backeberg, debido a que no acepta que las variaciones locales sean suficientes para que se consideren subespecies distintas. Posteriormente es tratada por Anderson (2001) como Neoraimondia arequipensis (Meyen) Backeberg, aunque acepta la existencia de dos subespecies que se diferencian en el color de la flor, número de costillas, tamaño y la localidad de origen. Finalmente, Ostolaza et al. (2005) la tratan como Neoraimondia arequipensis subsp. roseiflora (Werdermann \& Backeberg) Ostolaza, caracterizada por tener 4 a 6 costillas, flores de color rosado y que se distribuye en el valle de Fortaleza, al sur de Ancash, todos los valles del Dpto. de Lima hasta Pisco, Dpto. de Ica (Castro, 2006) (Figuras 1 y 2).

\section{Descripción botánica}

Planta columnar robusta de $1.3-4 \mathrm{~m}$ de altura, ramificada desde la base, formando grupos de 4-20 tallos. Tallo terete de 15-20 cm de diámetro, con 4 -8 costillas. Areolas grandes marrón oscuro, afelpadas, de crecimiento continuo, de 1-26 X 2-3 cm. Espinas color gris de 6-25 cm, en grupos de 6-21 por areola. Flores actinomorfas, infundibuliformes, rosadas a rojo grosella, sobre las areolas, de 4.7-6 cm y perianto de 1.7-2.2 cm de diámetro, con aproximadamente 40-50 tépalos; los sepaloides lanceolado-apiculado con el ápice negro y los petaloides ligulado-rotundos. El tubo del receptáculo de 3.5 -4.5 x 1.1-1.5 cm y la cámara nectarial de 1.1-1.4 x 0.5-0.7 cm. Androceo con aproximadamente 350 estambres epipétalos de 1.1-1.8 cm; las anteras bitécicas amarillas de $0.2-0.3 \mathrm{~cm}$ y los filamentos color marfil de $0.9-1.5 \mathrm{~cm}$. Pericarpelo de 1.3-1.8 x 1.1-1.6 cm, cubierto con pequeñas brácteas a manera de escamas, pelos rizados y pequeñas espinas.
Gineceo sincárpico de 3.9-5.4 cm, ovario ínfero de 0.5-1.3 x 0.4-0.8 cm, con 10-12 carpelos y numerosos óvulos de placentación parietal. Estilo de 2-2.5 cm y estigma lobulado de $0.5 \mathrm{~cm}$, con 10-15 lóbulos verdes. Fruto baya de color rojo a guinda, globular de 3.5-4 cm de diámetro, cubierto de areolas redondas con pelos y espinas pequeñas. Funículo ariloso desarrollado, blanco rosado. Semillas numerosas (1000-2000) reniforme-ovadas, negras o marrones, de $0.15 \times 0.1 \mathrm{~cm}$ y la testa reticulada-foveolada (Novoa, 2005; Castro, 2006; Hunt, 2006) (Figura 3).

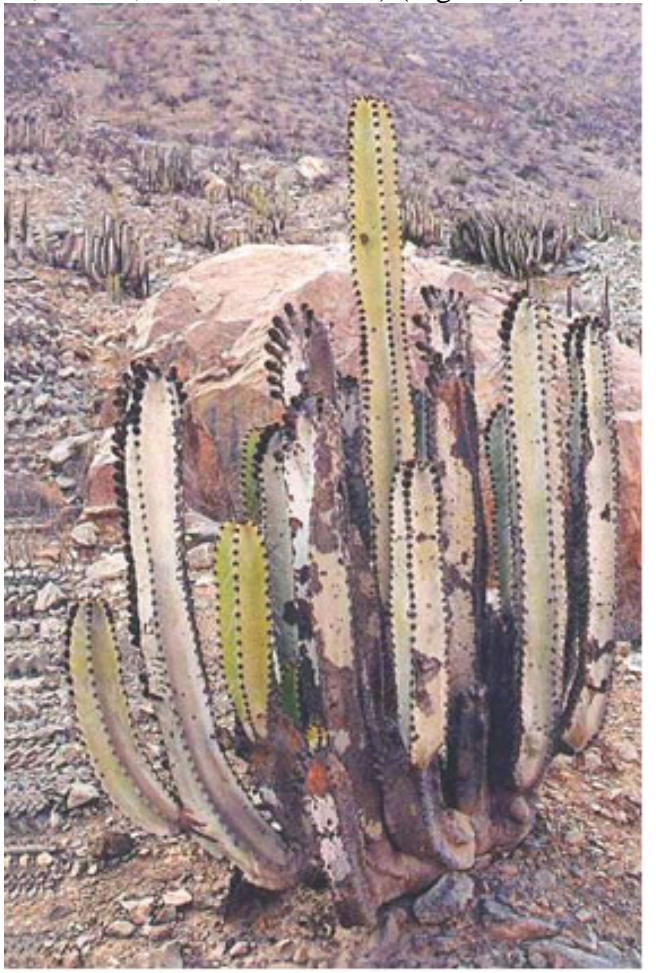

Figura 1. Neoraimondia arequipensis subsp. roseiflora (Werdermann \& Backeberg) Ostolaza. Cerro Umarcata, Santa Rosa de Quives. Cuenca baja del río Chillón (Novoa et al., 2003).

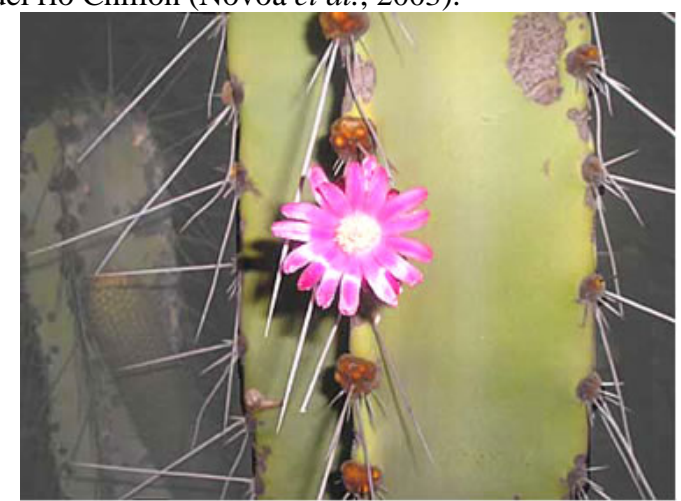

Figura 2. Neoraimondia arequipensis subsp. roseiflora (Werdermann \& Backeberg) Ostolaza, en floración. Cerro Umarcata, Santa Rosa de Quives. Cuenca baja del río Chillón (Foto: Redolfi, 2004). 


\section{Distribución}

$N$. arequipensis subsp. roseiflora tiene una amplia distribución a través de los Andes Peruanos, en los Departamentos de Ancash, Lima e Ica. Está presente en el valle del río Fortaleza (límite norte) en Ancash, en los valles de Supe, Huaura, Chancay, Chillón, Rímac, Lurín, Mala, Chilca, Omas y Cañete, en Lima y Chincha y Pisco (límite sur) en Ica. Altitudinalmente, se distribuye entre los 500 y 1700 msnm, según los valles (Brako \& Zarucchi, 1993; Ostolaza, 2003; Ostolaza et al., 1985, 2003, 2005, 2006, 2007; Hunt, 2006) (Figura 4). Se considera una especie endémica (Arakaki et al., 2006).

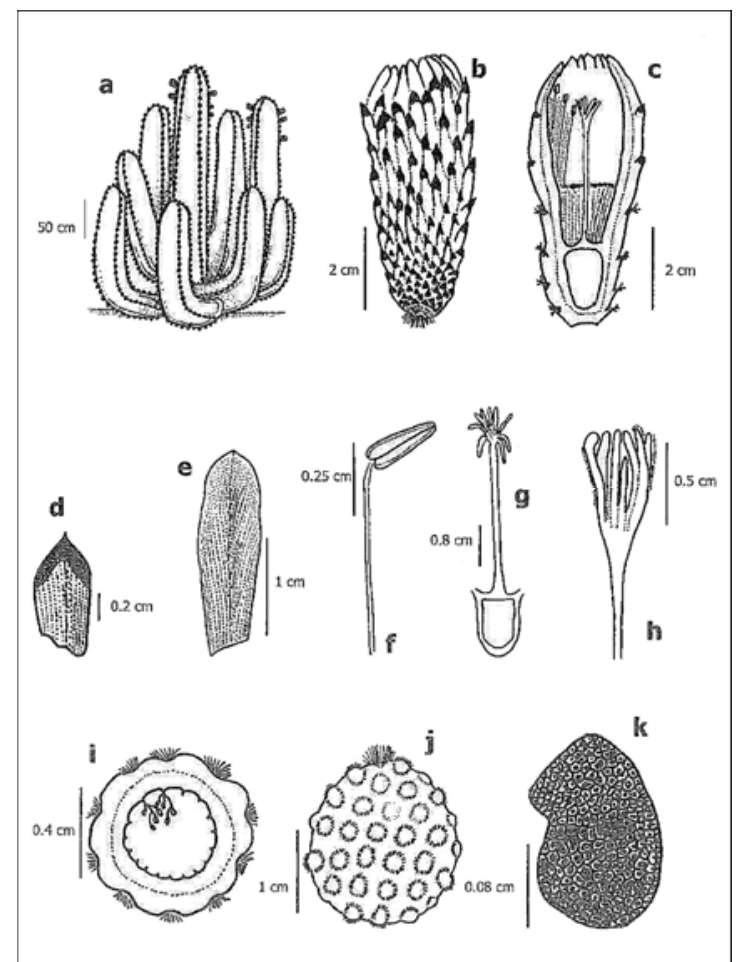

Figura 3. Neoraimondia arequipensis subsp. roseiflora (Werdermann \& Backeberg) Ostolaza. a. Hábito; b. Flor; c. Sección longitudinal de la flor; d. Tépalo sepaloide; e. Tépalo petaloide; f. Estambre; g. Gineceo; h. Estigma; i. Sección transversal del ovario; j. Fruto; k. Semilla (Castro, 2006).

\section{Categorización}

De acuerdo a la Categorización de la IUCN (2001) y debido a la presencia de miles de individuos en los valles de Ancash, Lima e Ica sin signos de alteración, la especie puede considerarse como de Preocupación Menor (LC) (Ostolaza, 2003; Ostolaza et al. 2006)

\section{Zona de Vida}

$N$. arequipensis subsp. roseiflora se ubica principalmente en la zona de vida Matorral Desértico Sub - Tropical (md-ST), distribuido entre los 800 y 2100 msnm. Esta zona presenta un clima árido y semicálido. La precipitación promedio anual es mayor a los $125 \mathrm{~mm}$ y la temperatura promedio anual es $18^{\circ} \mathrm{C}$. La vegetación natural está constituida en la parte más baja del piso por cactáceas columnares y vegetación reducida (ONERN, 1976; Weberbauer, 1945).

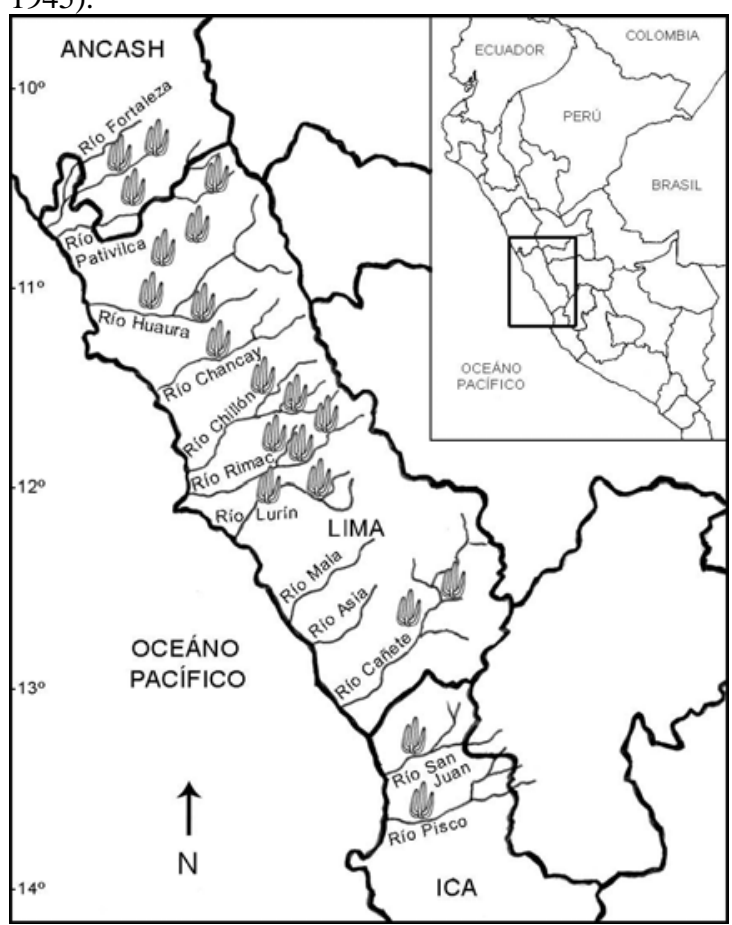

Figura 4. Distribución de Neoraimondia arequipensis subsp. roseiflora en el Perú (Figura: Ceroni, 2007).

\section{Adaptaciones morfo-anatómicas}

$N$. arequipensis subsp. roseiflora presenta lo que Anderson (2001) describe como un hábito arbustivo (varias ramas que salen del nivel del suelo) y columnar (tallo erecto en forma de cilindro, ramificado o no, segmentado o no, corto o largo). Los tallos son generalmente verdes, en estadios juveniles y conforme el tallo va ganando edad se lignifica y se cubre de una gruesa cutícula cerosa (Barthlott \& Hunt, 1993). Debido a que estas plantas colonizan principalmente ambientes xéricos, los tallos son suculentos, de manera que pueden almacenar agua. Sin embargo, al aumentar su volumen tienden a aumentar la superficie por lo que el área de transpiración aumenta también y a la larga esto puede ser peligroso para la planta (Mauseth, 2000). El tallo presenta hendiduras longitudinales, las cuales producen la proyección de crestas llamadas “costillas”, las cuales varían en número y tamaño (Barthlott \& Hunt, 1993). Pocas costillas muy grandes, como el caso de $N$. arequipensis subsp. roseiflora actúan como hojas y no permiten una significativa variación del volumen, por lo que se espera que estas plantas se presenten en ambientes más húmedos. Por otro lado, las costillas pueden interactuar con factores ambientales como el viento y 
la radiación (Mauseth, 2000). Las areolas son elementos semejantes a las yemas existentes en los tallos de las dicotiledóneas. Se originan a partir de una yema, en las axilas de las hojas, encima de los tubérculos de las costillas o en el borde de ellos; constituyen una ramificación lateral del cuerpo de la planta (Hoffmann, 1989). Estas ramas tienen nudos y entrenudos, los cuales son muy cortos, por lo que se presentan como una masa de nudos, espinas y estructuras reproductivas; son radiales o bilaterales, en algunos géneros elongadas, bipartidas o divididas, con tricomas, espinas, cerdas y flores en algunos casos (Barthlott \& Hunt, 1993; Anderson, 2001). La disposición de las yemas de las hojas y de las areolas constituye una filotáxis alterna (Barthlott \& Hunt, 1993).

Sólo en el caso de $N$. arequipensis subsp. roseiflora las areolas pueden continuar creciendo y produciendo tricomas y espinas, aun en tallos viejos; en pocos casos la areola se constituye en una estructura muy prolongada que produce flores anualmente (Anderson, 2001). Las espinas están presentes en casi todos los géneros, al menos durante la fase de germinación y establecimiento. Estas, crecen de las areolas y son las verdaderas hojas modificadas; se presentan en variedad de formas, colores, número y tamaño, las cuales varían además dependiendo de la edad de la planta (Barthlott \& Hunt, 1993; Gibson, 1979). Las espinas cumplen diversas funciones, siendo las más evidente la protección contra los herbívoros, sin embargo, también sirven como camuflaje, reflejan la luz solar sombreando el tallo, reducen la pérdida de agua por evaporación, reducen el movimiento del aire alrededor del tallo, captan la humedad de la niebla y del rocío, la cual cae al suelo que rodea a la planta y en algunos casos sirven como medios de dispersión vegetativa (Anderson, 2001).

La organización de los tejidos de las cactáceas es muy similar a la de cualquier dicotiledónea con hojas laminares. La epidermis del tallo está formada por una capa simple de células de pared celular primaria delgada, altamente cutinizada $\mathrm{y}$ en algunos casos acompañada de varias capas de cera. Esta cera es responsable del color blanquecino y azulino de algunos tallos. Los estomas son típicamente paracíticos, es decir, las células subsidiarias están alineadas paralelamente a las células guardianas. Debajo de la epidermis está la hipodermis, formada por varias capas de células de paredes celulares engrosadas, la cual da resistencia mecánica al tallo y en algunas especies se acumulan cristales de oxalato de calcio. La corteza está formada principalmente por células que realizan diversas funciones: clorénquima, que realiza la fotosíntesis y parénquima, que almacena agua. Los haces conductores están compuestos por xilema y floema en disposición colateral; en la parte central se ubica una pequeña médula, la cual puede tener pequeños haces conductores (Anderson, 2001).

La madera de los tallos es especializada; presenta xilema compuesto por vasos anchos y cortos, las fibras permanecen vivas y son estrechas, el cambium vascular produce gran cantidad de células parenquimáticas, por lo que se observan grandes radios de parénquima entre el tejido vascular; algunas especies presentan traqueidas anchas sin punteaduras junto a los vasos verdaderos (Mauseth \& Plemons, 1997). Esta madera no solo cumple la función de conducción sino que además cumple una función de soporte, afectando la forma general de la planta (Terrazas \& Mauseth, 2002).

\section{Fenología}

Los estudios de fenología son de suma importancia no sólo en la comprensión de la dinámica de las comunidades vegetales, sino también como un indicador de la respuesta de estos organismos a las condiciones climáticas y edáficas de una zona en particular (Fournier, 1969). Los estados fenológicos de una especie pueden afectar a la planta a múltiples niveles, incluyendo los sucesos reproductivos individuales de la planta, interacciones con otros organismos (afectando la variación y abundancia espacio-temporal de polinizadores y dispersores), dinámicas poblacionales vegetales y el funcionamiento del ecosistema (Bronstein, 1995; McIntosh, 2002). Los estudios acerca de la fenología de las cactáceas son muy escasos o han sido considerados en otros procesos ecológicos tales como la polinización por murciélagos y polillas y dispersión por murciélagos (Sahley, 1996; Valiente et al., 1996, 1997; Fleming \& Holland, 1998; Rojas et al., 1999; Holland \& Fleming, 1999, 2002; De Viana et al., 2001; Molina et al., 2004).

$N$. arequipensis subsp. roseiflora es una especie que presenta una gran irregularidad en su fenología durante el año, y es posible observar hasta cuatro estadios fenológicos diferentes en una sola planta. Estudios fenológicos realizados en el valle del río Chillón, en el cerro Umarcata, entre el 2003 y 2004 (Novoa, 2005; Novoa et al., 2005 a) constataron la presencia de dos variables fenológicas bien marcadas: floración y fructificación (Figuras 5 y 6). Cada una de estas se encuentra formada por cuatro estadios fenológicos. La floración se desarrolla en los meses de Noviembre a Abril y la fructificación en Mayo y Octubre. El patrón hipotético propuesto para la fenología de $N$. arequipensis es: a Enero le corresponde el estadio fenológico de flor marchita; a Febrero y Marzo el de botón abortado; al mes de Abril, el estadio de floración total; Mayo y Junio, el estadio fenológico de fruto inmaduro o - verde -; Junio también el mes correspondiente al estadio vegetativo; Julio al de dispersión de semillas mediante la dehiscencia del fruto; Agosto y Setiembre de fruto maduro; Octubre de frutos apareciendo; a Noviembre 
le corresponde el estadio de botón floral y Diciembre nuevamente el estadio de botón abortado.

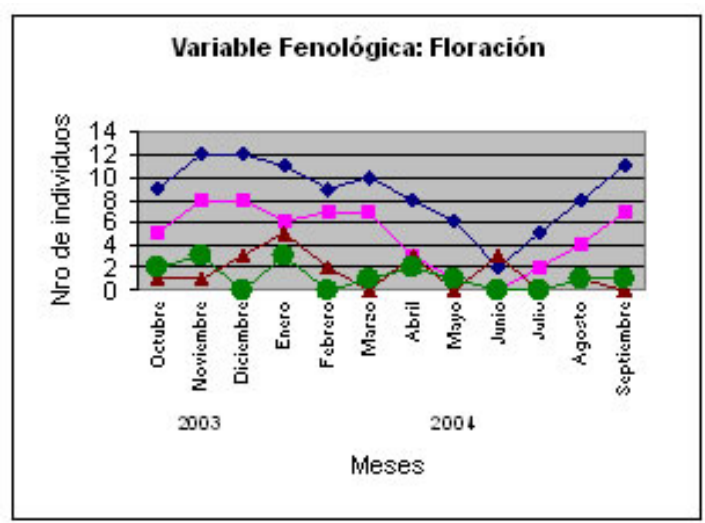

Figura 5. Fenograma de las etapas de la floración de Neoraimondia arequipensis subsp. roseiflora. Octubre 2003 - Setiembre 2004. (Botones florales $\rightarrow$ Botones abortados $\rightarrow$, Floración total $\rightarrow$, Flores marchitas $\rightarrow-$ ) (Novoa et al., 2005 a).

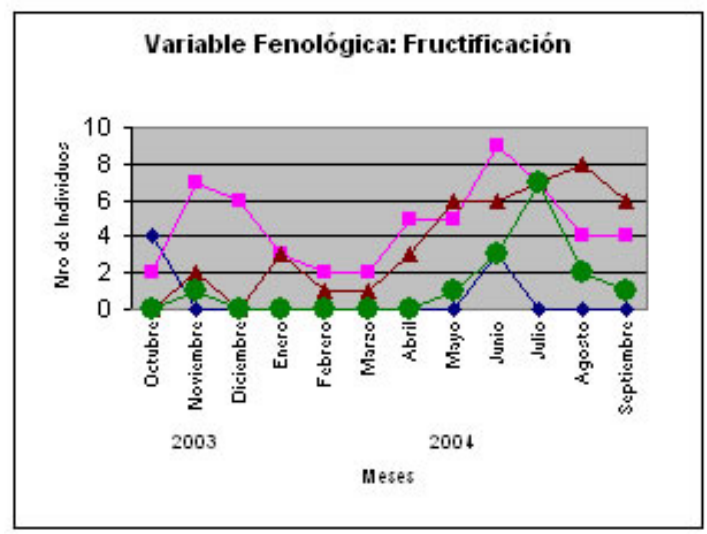

Figura 6. Fenograma de las etapas de la fructificación de Neoraimondia arequipensis subsp. roseiflora. Octubre 2003 - Setiembre 2004. (Frutos apareciendo $\rightarrow$, Frutos inmaduros - , Frutos maduros $\rightarrow$, Dispersión $\rightarrow-$ ) (Novoa et al., 2005 a)

Cabe resaltar que lo más notorio de esta secuencia estacional es la constante producción de botones florales en gran parte del año, que podría hallar su explicación en las características morfológicas de la especie. $N$. arequipensis es un cactus que se ramifica desde la base, sus tallos no son articulados lo cual significa que su reproducción vegetativa es muy poco probable. Esta característica obliga a la planta a tener que reproducirse sexualmente $\mathrm{y}$ generar nuevas plántulas mediante la germinación de sus semillas. Otra característica a tomar en cuenta es el crecimiento elongado de las areolas, las plantas siempre muestran el dilema de distribuir los escasos recursos entre la producción de estructuras florales o estructuras vegetativas. La elongación de las areolas se muestra como una buena alternativa para reducir el costo de producción de flores al mínimo, además de que cada areola puede producir una o más flores año tras año a diferencia de la mayoría de los cactus que producen una sola flor al año (Mauseth \& Kiesling, 1997, Mauseth et al., 2002). Esto último, sumado al número de ramas y a la producción de areolas generadoras de estructuras reproductivas en potencia, hace que el evento de producción de botones florales sea masivo y significativo a lo largo del año (Novoa, 2005; Novoa et al., 2005 a).

\section{Interacciones}

Las cactáceas cumplen un rol importante en los ecosistemas constituyendo un elemento esencial en el paisaje, gracias al sistema radicular amplio $\mathrm{y}$ superficial que forma una malla que interviene en los procesos de erosión y desertificación de los suelos y puesto que las raíces poseen pelos absorbentes caducos se constituyen como una fuente continua de materia orgánica que se incorpora al suelo (Magallanes, 1997). Los cactus interaccionan con diversos organismos en el ecosistema. Frente a las inclemencias del medio ambiente desarrollan estrechas relaciones con otras especies vegetales denominadas "plantas nodrizas" que ofrecen las condiciones de humedad y temperatura para el establecimiento de nuevas plántulas (Turner et al., 1966; Valiente et al., 1991a b; Valiente \& Ezcurra, 1991; Nobel, 1998; De Viana et al., 2000) y algunas veces crean las condiciones necesarias para el establecimiento de otros cactus (McAuliffe, 1984). Los tallos son la única fuente de fibra y agua para los animales silvestres en los ecosistemas desérticos (Nobel, 1998; Gibson \& Rundel, 2001; Márquez et al., 2003). Algunas aves utilizan a los tallos de los cactus como un inmejorable refugio frente a potenciales depredadores haciendo sus nidos sobre e incluso dentro de la planta (McAuliffe \& Hendricks, 1988; Rivera \& Rodriguez, 1998). Las flores y frutos son fuente de alimento de numerosas aves como colibríes y palomas, mamíferos como roedores y murciélagos, destacándose de estos grupos dos especies que se encuentran en peligro crítico de extinción en el Perú como lo son la pava aliblanca (Penelope albipennis) y el murciélago longirostro peruano (Platalina genovesium) (Lerner et al., 2003; Sahley, 1996). Asimismo, una gran gama de insectos visitan estas plantas, de los cuales se destacan las especies de los ordenes Lepidoptera, Diptera e Hymenoptera (Keopcke, 1973; Valiente et al., 1996; Valiente et al., 1997; Fleming \& Holland, 1998; Fleming et al., 2001; Godínez et al., 2002; Holland \& Fleming, 1999, 2002; Scobell \& Scout, 2002).

$N$. arequipensis subsp. roseiflora como elemento dominante en ecosistemas áridos en los Andes del Perú tiene una relación muy estrecha con los componentes del medio ambiente biótico y abiótico. Esta relación se manifiesta a través de una serie de 
interacciones tanto son el suelo como a través del nodricismo, la polinización, la dispersión, así como con la entomofauna circundante (Figura 7).

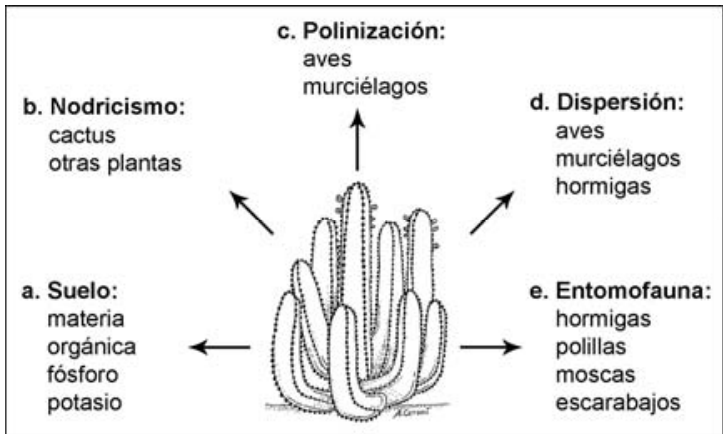

Figura 7. Interacciones de Neoraimondia arequipensis subsp. roseiflora con el ecosistema (Figura: Ceroni, 2007).

\section{Suelo}

$N$. arequipensis subsp. roseiflora como especie xerofítica está adaptada a suelos muy pobres. Estudios realizados en cuanto a la relación de la diversidad y densidad de cactáceas en el valle del río Chillón, en el cerro Umarcata y la quebrada Orobel, con los factores edáficos (Teixeira et al., 2004; Teixeira, 2006), han demostrado que la densidad de esta especie tiene una relación significativa con la materia orgánica del suelo, siendo esta una relación exponencial $\left(\mathrm{R}^{2}=\right.$ 0.397), en donde al aumentar el porcentaje de materia orgánica disminuye la densidad, mientras que en cuanto a la concentración de fósforo y potasio presentan también una relación relativamente significativa, exponencial en el caso del fósforo $\left(\mathrm{R}^{2}=\right.$ 0.4832) y polinomial de segundo grado en el caso del potasio $\left(\mathrm{R}^{2}=0.254\right)$, en donde las altas concentraciones de estos macronutrientes son un factor limitante para la densidad del cactus (Figuras 8, 9 y 10). El hecho que la materia orgánica no sea utilizada totalmente por las cactáceas en estas zonas de estudio pueda deberse a que, probablemente, gran parte del porcentaje de materia orgánica no se encuentre en forma disponible para las plantas por estar en proceso de mineralización. Asimismo, en cuanto a la relación con el fósforo y el potasio, se encontró que la máxima cantidad de cactáceas estaba determinada por un rango de fósforo entre 20 a 30 ppm y de potasio entre 300 a 450 ppm. Cuando las concentraciones de fósforo y potasio están fuera de estos rangos la densidad de cactáceas disminuye, indicando que estos macronutrientes estarían actuando como factores limitantes ecológicos (Sarmiento, 1984).

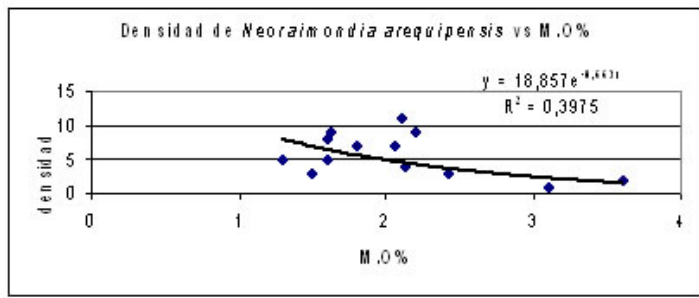

Figura 8. Correlación entre el porcentaje de materia orgánica (\%M.O.) y la densidad de Neoraimondia arequipensis subsp. roseiflora (Teixeira, 2006).

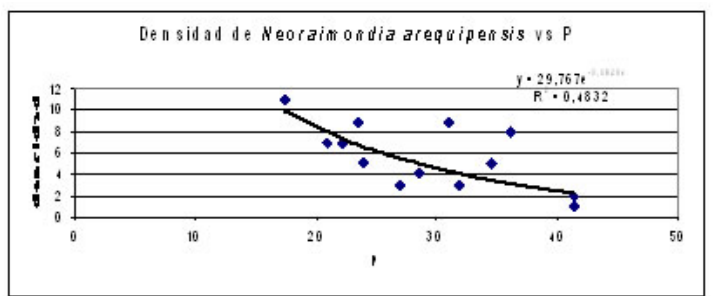

Figura 9. Correlación entre la concentración de fósforo (ppm) y la densidad de Neoraimondia arequipensis subsp. roseiflora (Teixeira, 2006).

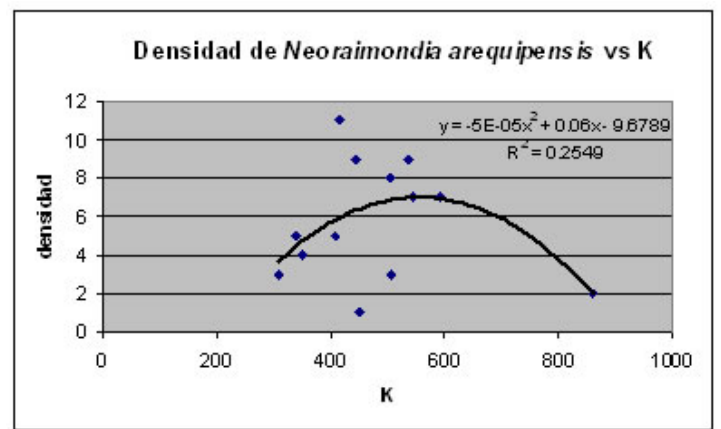

Figura 10. Correlación entre la concentración de potasio (ppm) y la densidad de Neoraimondia arequipensis subsp. roseiflora (Teixeira, 2006).

Es decir, que por encima de ciertos rangos la planta ya no los utiliza. Estos factores pueden producir un cambio en la densidad promedio o en la densidad equilibrio de la comunidad (Krebs, 2001). Un análisis de componentes principales para estas dos zonas de estudio mostró que los componentes del suelo, como el porcentaje de materia orgánica y disponibilidad de fósforo y potasio tienen una relación estrecha tanto con la densidad total de cactáceas como con la densidad individual de cada una de las especies. Para el caso de $N$. arequipensis subsp. roseiflora, el análisis demuestra que esta especie posee una relación relativamente estrecha y positiva con el Primer componente de 0.740. Sin embargo, esta especie en la quebrada Orobel, no muestra relaciones significativas con ninguno de los componentes de suelo. Esto puede deberse a que en esta zona en particular, los nutrientes del suelo no son el factor que determina la densidad o 
comportamiento de esta especie, siendo las relaciones bióticas las regulatorias del comportamiento de la comunidad (Teixeira, 2006).

En cuanto a la densidad, se encontró que esta es mayor en el cerro Umarcata, llegando hasta 11 individuos por $15 \mathrm{~m}^{2}$, mientras que en la quebrada Oropel lo máximo es de 10 individuos por $15 \mathrm{~m}^{2}$. Sin embargo, en esta última zona la densidad de esta especie es menos variable es decir más equitativa en toda la zona, mientras que en el cerro Umarcata, se nota claramente núcleos de alta densidad en diferentes partes, indicando una distribución más variable (Figura 11 a y b).
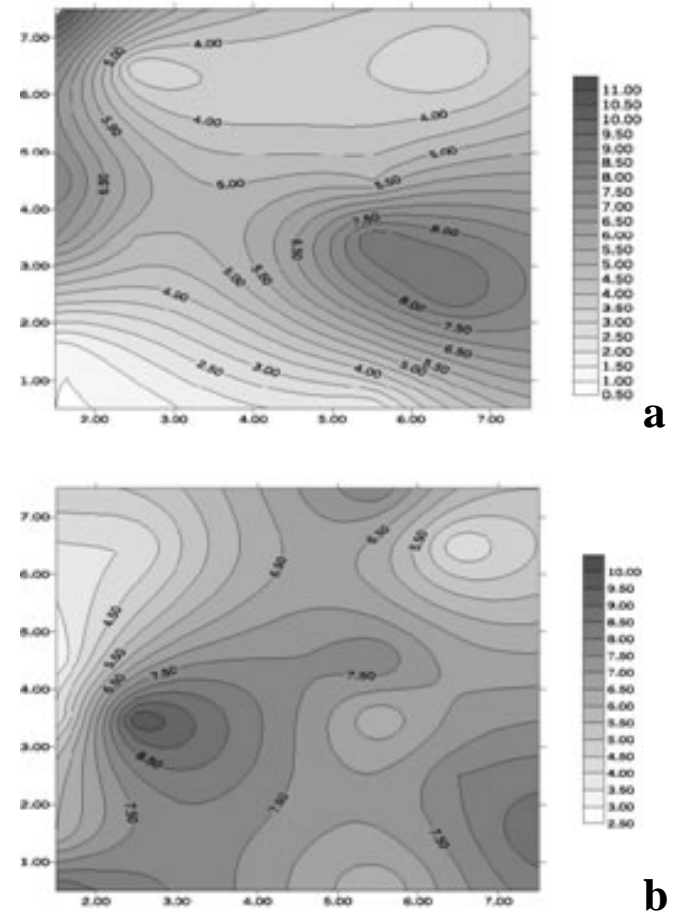

Figura 11. Isolíneas de densidad de Neoraimondia arequipensis subsp. roseiflora en (a) cerro Umarcata y (b) quebrada Oropel (Teixeira, 2006).

\section{Nodricismo}

Al ser Neoraimondia arequipensis subsp. roseiflora una especie de alta densidad, participa en una de las interacciones benéficas entre las plantas de ambientes áridos, llamada nodricismo, actuando como nodriza (Figura 12). Una planta nodriza provee protección a sus plántulas o a las de otra especie, de estrés por calor, deficiencia de agua o nutrientes y herbivoría (Leirana \& Parra, 1999). El fenómeno puede deberse a que las cactáceas son plantas suculentas con metabolismo CAM, las cuales no pueden regular su temperatura a través de la transpiración. Así, la protección del estado de plántula contra la radiación podría ser crucial en su supervivencia (Valiente et al., 1991 a). Por otro lado, la acumulación de semillas tiende a ser mayor al pie de las plantas en comparación con ambientes abiertos; también puede deberse a que las semillas son llevadas a estos lugares por animales, agua o el viento. Las semillas y plántulas camufladas por otras plantas tienden a sobrevivir en mayor número que las plantas que crecen en áreas abiertas (Valiente \& Ezcurra, 1991).

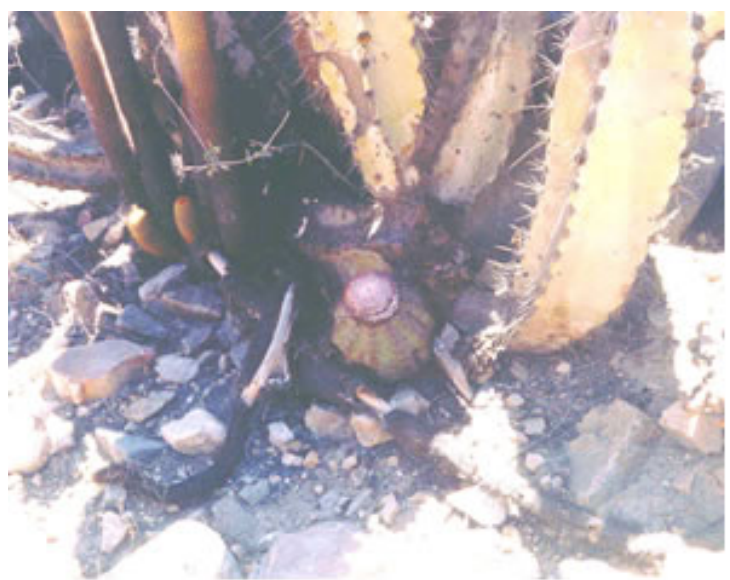

Figura 12. Haageocereus pseudomelanostele subsp. aureispinus y Melocactus peruvianus creciendo bajo una Neoraimondia arequipensis subsp. roseiflora en el cerro Umarcata (Foto: Ceroni, 2007).

El patrón de establecimiento de las plantas se debe a la protección de los jóvenes cactus por otras plantas u otros cactus que forman un dosel. Este, los protege del exceso de radiación solar, pérdida de agua excesiva, congelamiento, depredación o daño mecánico, temperaturas cambiantes del suelo, exceso o escasez de nutrientes en el suelo, entre otros (Valiente et al., 1991 b; Valiente \& Ezcurra, 1991). El establecimiento de plántulas de cactus bajo otras plantas u otros cactus puede eventualmente afectar el efecto nodriza. Esto quiere decir que la planta que utiliza una nodriza puede competir con ella o terminar matándola incluso (Valiente et al., 1991 b).

El crecimiento bajo plantas nodrizas afecta también la distribución de los cactus, haciendo que estos presenten una distribución agregada, lo que puede representar una interacción relevante entre el cactus y su nodriza en las comunidades de zonas áridas (Leirana \& Parra, 1999). El entendimiento de las dinámicas en el desarrollo temprano de las semillas y plántulas es de vital importancia para entender la dinámica de las poblaciones y la estructura de la comunidad del desierto (Valiente \& Ezcurra, 1991).

Estudios realizados en la comunidad de cactáceas en el cerro Umarcata y en la quebrada Orobel demuestran que si bien es cierto que cada una de las especies de cactáceas presentan patrones de distribución diferentes, también es posible encontrar relaciones entre las especies según su abundancia y su 
distribución espacial (Teixeira, 2006). Al observar las isolíneas de densidad de Neoraimondia arequipensis subsp. roseiflora, en el cerro Umarcata (Figura 11a) se encuentra un núcleo de alta densidad a la derecha de la zona de estudio. Esta especie es la cactácea columnar más grande de la comunidad además de la más ramificada por lo que debajo de esta se albergan algunas de las otras especies de cactáceas como el Melocactus peruvianus o Mila nealeana, cuyos núcleos de alta densidad son mas o menos coincinentes con los de $N$. arequipensis subsp. roseiflora (Figura 13 a y b). Esta última actúa como nodriza, protegiendo a los cactus jóvenes durante su crecimiento, brindándoles sombra, protegiéndolos del viento y de los depredadores. Esta puede ser una de las causas por las que las cactáceas se distribuyen en forma aglomerada en el área, es decir formando asociaciones temporales (Teixeira, 2006).
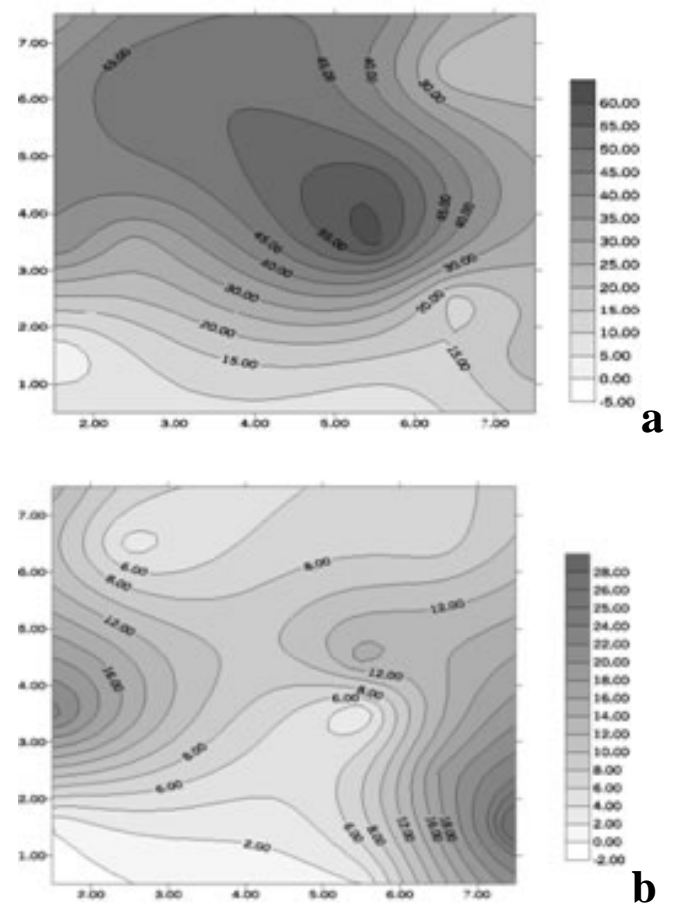

Figura 13. Isolíneas de densidad de (a) Melocactus peruvianus y (b) Mila nealeana en el cerro Umarcata (Teixeira, 2006).

Por otro lado, en la quebrada Oropel Neoraimondia arequipensis subsp. roseiflora tiene una distribución diferente que en el Cerro Umarcata, pero cumple también un papel determinante para la supervivencia de los cactus jóvenes de su especie como de las otras especies de la comunidad. En esta zona pareciera que se distribuye de forma casi regular o uniforme, salvo por un pequeño núcleo de alta densidad en la zona media izquierda (Figura 11b), por lo tanto, pareciera que estuviera cumpliendo una labor generalizada de nodricismo, siendo esta mucho más estrecha con Mila nealeana con la que comparten un pequeño núcleo de alta densidad en la zona baja derecha del área de estudio (Figura 14).

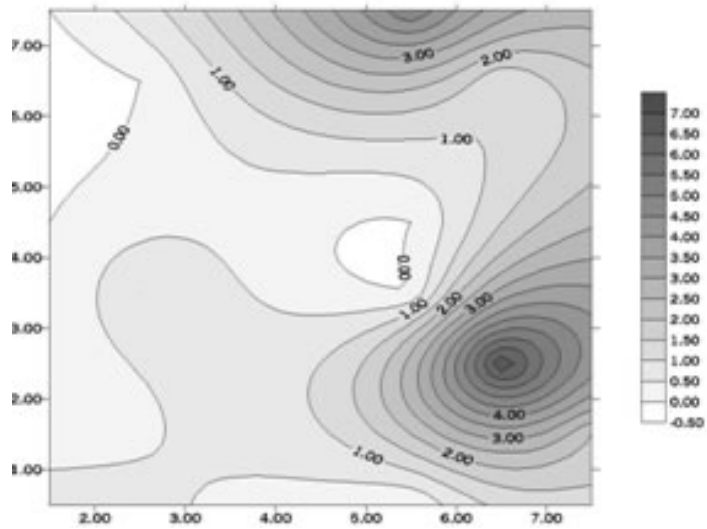

Figura 14. Isolíneas de densidad de Mila nealeana en la quebrada Orobel (Teixeira, 2006).

\section{Polinización y dispersión}

La polinización de plantas con flores por animales representa uno de los procesos más importantes de la biología reproductiva de cerca del $90 \%$ de las especies de angiospermas (Kearns et al., 1998) La familia Cactaceae es reconocida como un grupo de plantas fuertemente relacionada con la polinización por animales (Gibson \& Nobel, 1986).

Algunas referencias de estos estudios en $N$. arequipensis subsp. roseiflora son repetidos mediante observaciones ocasionales de Eastwood (2006) en $N$. arequipensis subsp. gigantea (Werderman \& Backeberg) Ostolaza en las zonas desérticas al margen de la carretera de Pacasmayo a Cajamarca, citando en esta oportunidad a la mariposa especialista Strymon jacqueline Nicolay \& Robbins 2005 (Lepidoptera: Lycaenidae: Eumaeini) alimentándose del néctar extrafloral del fruto y también visitando las flores. Además citan a avispas y moscas atraídas por el néctar extrafloral exudado, pero no observaron la presencia de hormigas en el cactus. En una exploración de otro hábitat, este autor refiere la presencia de hormigas obreras del género Crematogaster y algunos especímenes de Pseudomyrmex en nectarios extraflorales en $N$. arequipensis subsp. gigantea, pero sin especificar la ubicación de las estructuras extraflorales. Estas observaciones podría dar fundamento a la hipótesis propuesta que los nectarios extraflorales del cactus pueden propiciar la polinización (Ford \& Forde, 1976; Wäckers \& Bonifay, 2004).

La forma más común de dispersión de las semillas es a través de las aves, lo que se hace evidente debido a las características de los frutos (jugosos y dulces). También presentan una doble estrategia que involucra aves y hormigas: los frutos que no son comidos por 
las aves, caen de la planta al suelo y se secan, permitiendo la acción de las hormigas. Por otro lado, son comidos por mamíferos, ya sea que caigan al suelo o se mantengan unidos a la planta (Barthlott \& Hunt, 1993; Anderson, 2001).

\section{Entomofauna}

Los artrópodos, principalmente los insectos, se relacionan de diversas maneras con las plantas y si bien los cactus mantienen una relación más restringida debido a su estructura morfológica, Neoraimondia arequipensis subsp. roseiflora es la excepción en el ecosistema de zonas áridas en el valle del río Chillón, lugar en donde se han realizado las principales investigaciones de estas interacciones. La primera observación de un insecto relacionado a $N$. arequipensis subsp. roseiflora fue realizada por Ostolaza et al. (1985) al citar la presencia de grandes y negras hormigas en los brotes florales, flores abiertas y frutos; agregando posteriormente información al referirse a las flores: "se cree que ciertas hormigas negras sean las polinizadoras” (Ostolaza, 1987).

El inicio del estudio de la hormiga negra en $N$. arequipensis subsp. roseiflora se debió a la hipótesis según la cual este insecto sería un indicador de nectarios externos al botón floral en base a las observaciones preliminares realizadas en el cerro Umarcata (Figura 15). Esta hipótesis fue demostrada por Novoa et al. (2003), quienes determinaron que la hormiga negra Camponotus sp. forrajea únicamente en el exterior de los botones florales de este cactus, en una relación altamente significativa, en el $100 \%$ de las plantas con botones florales y no se presenta en ninguna de las otras siete especies de la comunidad de Cactáceas: Austrocylindropuntia pachypus (Shumann) Backeberg , Mila nealeana Backeberg, Haageocereus acranthus (Vaupel) Backeberg, H. pseudomelanostele subsp. aureispinus (Rauh \& Backeberg) Ostolaza, Cleistocactus acanthurus subsp. faustianus (Backeberg) Ostolaza, Melocactus peruvianus Vaupel y Espostoa melanostele (Vaupel) Borg.

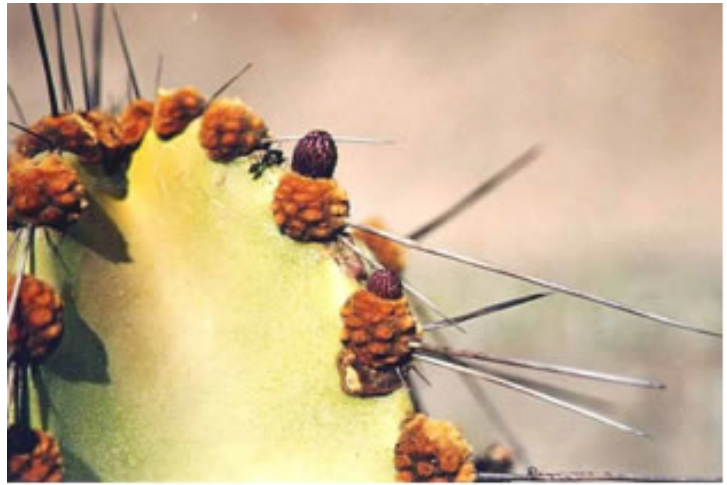

Figura 15. Hormiga Camponotus sp. forrajeando botones florales de Neoraimondia arequipensis subsp. roseiflora (Novoa et al. , 2003).
Los resultados demostraron que la hormiga se alimenta de las sustancias azucaradas en la zona exterior de los botones florales en donde permanecía largo tiempo (hasta 20 minutos) y no formaba pistas al desplazarse por las ramas. La prueba de presión del gáster demostró gran abundancia de alimento líquido lo que confirmó la relación hormiga-nectarios extraflorales, caso muy frecuente en ecosistemas de zonas áridas (Pickett \& Clark, 1979; Blom \& Clark, 1980; Ruffener \& Clark, 1986; Pemberton, 1988; Oliveira et al., 1999).

Prosiguiendo los estudios de esta relación, Novoa et al. (2005 b y c) determinaron la secuencia estacional de forrajeo de Camponotus sp., que presenta un pico durante los meses de Marzo, Abril y Mayo con un punto máximo en el mes de Abril en respuesta al estadio fenológico de una nueva campaña de botones florales con una mayor concentración de néctar, más atractivos para la hormiga, así como temperaturas más cálidas $\left(24^{\circ} \mathrm{C}\right)$ y menos humedad (45\% HR) (Figura 16). El patrón de actividad diario de la hormiga es unimodal con un máximo entre las 6 y 7 pm y un mínimo entre las 6 y 7 am y depende principalmente de la permanencia del recurso alimenticio de su preferencia en el medio y los rangos óptimos de las diferentes variables ambientales, tal como mencionan otros autores para diversas especies de hormigas (Figura 17) (Cerdá \& Retana, 1988; Cerdá et al., 1989; Farji-Brener, 1993; Morris, 1997; Redolfi et al., 2003). La hormiga presentó mayor dinamismo durante las horas de la noche, lo que estaría relacionado a una mayor oferta de alimento del cactus, tal como ocurre en otros cactus de floración nocturna (Valiente et al., 1997; Holland \& Fleming, 1999; De Viana et al., 2001; Clark \& Molina, 2004; Molina et al., 2004).

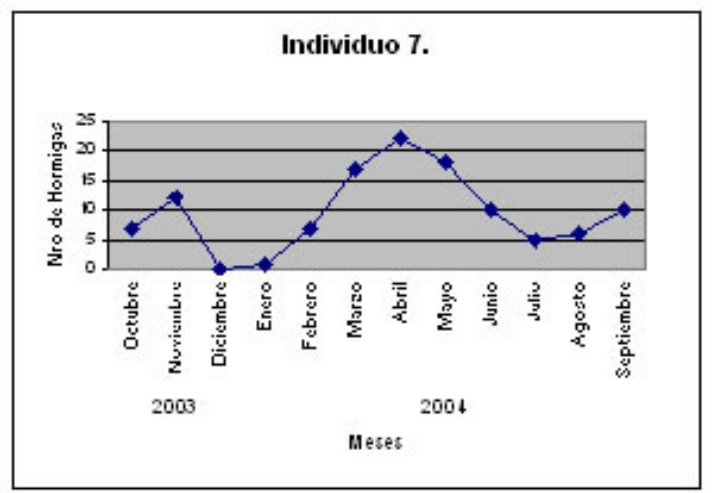

Figura 16. Secuencia estacional de la hormiga Camponotus sp. en el individuo 7 de Neoraimondia arequipensis subsp. roseiflora. Octubre 2003 Setiembre 2004 (Novoa, 2005). 


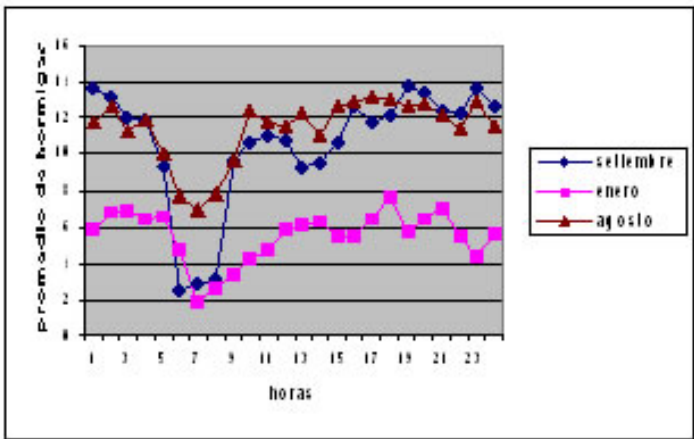

Figura 17. Patrón de actividad de la hormiga Camponotus sp. sobre Neoraimondia arequipensis subsp. roseiflora durante los días 20 de Setiembre del 2003; 31 de Enero del 2004 y 20 de Agosto del 2004 (Novoa et al., 2005 b).

La estrecha relación de Camponotus sp. durante la secuencia estacional en la oferta de alimento por el cactus a la hormiga y el comportamiento agresivo por parte de Camponotus sp. frente a insectos fitófagos (Diptera y Lepidoptera), hacen suponer que el néctar segregado podría ser la recompensa que reciben las hormigas por la protección frente a herbívoros, a pesar que la hormiga no presentó un comportamiento depredador. Numerosos estudios han demostrado que las hormigas son atraídas por el néctar que secretan las plantas y esta relación podría tener como finalidad la exclusión de fitófagos y un mayor acceso a nutrientes por aireación del suelo en el establecimiento de los nidos (Deuth, 1977; Inouye \& Taylor, 1979; Koptur, 1979; Pickett \& Clark, 1979; Schemske, 1980; Keeler, 1981; Stephenson, 1982; Horvitz \& Schemske, 1984; Barton, 1986; Davidson \& McKey, 1993; Wagner, 1997; Fonseca, 1999; Oliveira et al., 1999; Teixeira et al., 2004). Paralelamente a los estudios de Camponotus sp. se iniciaron las observaciones preliminares para determinar la presencia de otros artrópodos visitantes a la comunidad de cactus en el cerro Umarcata. Novoa et al. (2004), comprueban que $N$. arequipensis subsp. roseiflora es el eje principal de las interacciones aéreas en la comunidad de cactáceas, al observar la asidua visita en los botones florales y flores de dos especies de Diptera y una de Lepidoptera, Coleoptera, Thysanoptera y un Salticidae (Figura 18).

Esta comunidad de artrópodos presenta poblaciones más numerosas durante el mes de Enero, coincidente con mayores niveles de Humedad Relativa (98\%) y valores mínimos en el mes de Octubre, correspondiente con la época seca. Así también, Pacheco et al. (2004), en un estudio preliminar en los botones abortados en este cactus, el 95\% de los botones abortados presentaban insectos y/o indicios de la presencia de una especie de Coleoptera y/o una especie de Lepidoptera saprofíticos que podrían cumplir su ciclo biológico completo dentro del botón abortado. La gran cantidad de excrementos producidos por las poblaciones de estas dos especies, resultaría en un ingreso importante de nitrógeno al suelo. Estos autores también citan la interacción con tres especies de Hymenoptera parasitoides, aumentando el complejo sistema de la red trófica que mantiene $N$. arequipensis subsp. roseiflora.

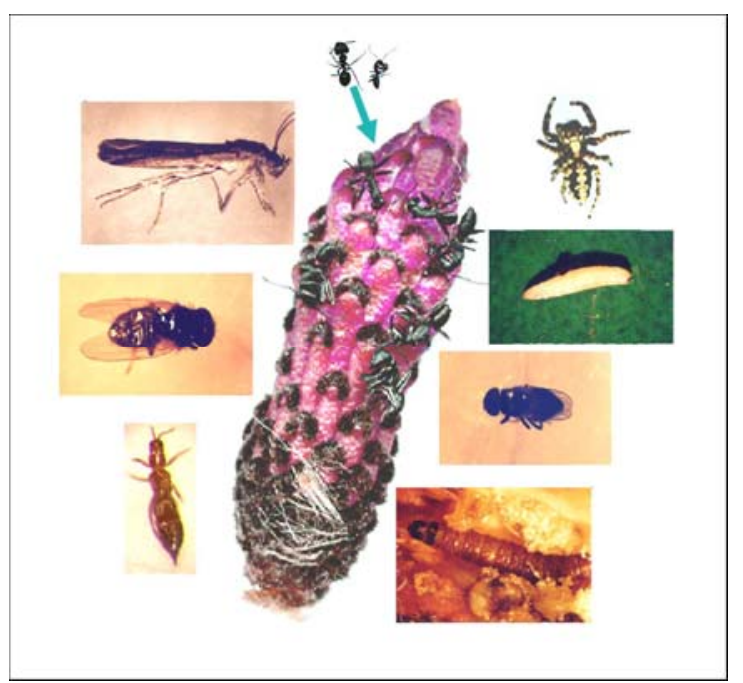

Figura 18. Artropodofauna (Lepidoptera, Diptera, Formicidae, Arachneae) que interacciona con el botón floral de Neoraimondia arequipensis subsp. roseiflora en el cerro Umarcata (Fotografías: Redolfi, 2004).

\section{Estado actual y futuros estudios en $N$. arequipensis subsp. roseiflora}

Estudios realizados en los últimos años en los valles de Lima y de manera especial en la cuenca baja del río Chillón han permitido conocer mejor algunos aspectos del cactus Neoraimondia arequipensis subsp. roseiflora y tener claro el papel que juega dentro de las comunidades de cactáceas en los ecosistemas áridos en los Andes en el Perú. Se ha podido ampliar la distribución más allá del departamento de Lima, hacia el norte hasta la quebrada del río Fortaleza en el departamento de Ancash y hacia el sur hasta el río Pisco en el departamento de Ica (Ostolaza et al., 2006). Sin embargo, como cactus endémico con una distribución restringida y debido al porte que tiene y al espacio necesario para su crecimiento y desarrollo, es importante resaltar la necesidad de conservar los ecosistemas en donde habita. Asimismo, a pesar de estar considerado como de Preocupación Menor (LC) (Ostolaza, 2003; Ostolaza et al., 2006), el rápido avance de la población humana está destruyendo su hábitat natural, como sucede actualmente en la quebrada California, en Chosica, lugar en donde también habita, lo cual podría poner en riesgo el estado de conservación de este cactus, que según Backeberg podría ser considerado la planta nacional a semejanza de lo que sucede en México o USA con 
otros céreos, ya que su hábitat se restringe exclusivamente al territorio nacional entre el nivel del mar y los dos mil metros de altitud en la vertiente occidental de los Andes (Ostolaza, 1987). Además, este es uno de los seis cactus utilizados para elaborar los palos de lluvia o palos de agua, artesanías peruanas de exportación confeccionadas principalmente con la madera seca de varias especies de cactáceas columnares. Aún cuando son otros los tres cactus, como Corryocactus brevistylus subsp. puquiensis (Rauh \& Backeberg) Ostolaza, Browningia candelaris (Meyen) Britton \& Rose y Weberbauerocereus rauhii Backeberg los más utilizados (Roque \& Ramírez, 2005) Neoraimondia arequipensis subsp. roseiflora también podría sufrir una presión en este sentido. Afortunadamente, todavía hay en Lima algunas zonas cuyo estado de conservación no es tan crítico, dentro de los cuales esta el cerro Umarcata, a la altura del Km 69 de la carretera Lima - Canta, en la cuenca baja del río Chillón, en donde se podría proponer planes de conservación in situ (Calderón, 2003).

La fenología del cactus $N$. arequipensis subsp. roseiflora y su estrecha relación con la hormiga Camponotus sp., quien visita sólo a esta especie de cactus de la comunidad de cactáceas, es otro de los aspectos que se ha logrado determinar (Novoa et al., 2003; Novoa, 2005; Novoa et al., 2005 a, b y c). Actualmente, como parte de estudios referentes a la germinación de semillas de los cactus del cerro Umarcata, con fines de conservación ex situ, se viene realizando más registros fenológicos incluyendo a $N$. arequipensis subsp. roseiflora lo cual permitirá tener mayor información de este evento tan importante.

Otras de las interacciones de $N$. arequipensis subsp. roseiflora que merecen mayores estudios es con respecto a sus relaciones de nodricismo con otros cactus o plantas de la comunidad de cactáceas. Aún cuando se ha podido inferir acerca del papel de planta nodriza que puede tener este cactus con respecto a otros más pequeños como Melocactus peruvianus y Mila nealeana, a partir de los estudios de diversidad y densidad de cactáceas (Teixeira, 2006) o de la importancia de la sombra que pueden dar estos cactus de gran porte para la supervivencia de plántulas de cactáceas de menor porte como Melocactus peruvianus y Haageocereus pseudomelanostele subsp. aureispinus (Castro et al., 2006), es necesario realizar futuros trabajos que nos permitan conocer la importancia de esta interacción en la dinámica del banco de semillas, así como también la relación que tiene este cactus con las especies herbáceas, ya que en la época húmeda de los valles costeros, lo más probable es que este actúa también como nodriza dentro de la dinámica de la comunidad vegetal en estos ecosistemas áridos.

Debido a la importancia de las interacciones de $N$. arequipensis subsp. roseiflora con la entomofauna, actualmente se están realizando estudios de la distribución y actividad de los hormigueros para complementar la dinámica poblacional de la hormiga negra Camponotus sp. Así mismo, el Proyecto tiene un gran avance en la investigación de las interacciones insectos-botones florales abortados.

Mayores estudios de entomofauna, nodricismo, vegetación acompañante, así como de polinización y de dispersión de $N$. arequipensis subsp. roseiflora están contemplados y canalizados en investigaciones sistematizadas dentro del Proyecto "Taxonomía, Ecología y Conservación Ex Situ de las Cactáceas de Lima”.

Por lo estudiado hasta el momento, la conservación de especies endémicas como Neoraimondia arequipensis subsp. roseiflora es de suma importancia para el buen mantenimiento y conservación de ecosistemas áridos, pero ello depende de la responsabilidad tanto de los diferentes agentes estatales y privados encargados del manejo del medio ambiente. En ese sentido, sería conveniente crear un área natural de conservación en estas comunidades de cactáceas en la cuenca baja del río Chillón, que involucre a $N$. arequipensis subsp. roseiflora como el eje principal del ecosistema, así como toda la comunidad vegetal asociada a este cactus. Dicha área de conservación permitirá no sólo proteger la comunidad, sino también realizar futuras investigaciones que permitan, conocer mejor la dinámica de estas poblaciones y la estructura de la comunidad del desierto.

\section{Agradecimientos}

Los autores expresan al Dr. Carlos Ostolaza Nano, especialista en cactáceas peruanas, por la revisión del artículo y sugerencias acertadas para mejorar el texto. El Proyecto “Taxonomía, Ecología y Conservación Ex Situ de las Cactáceas de Lima” está siendo financiado por el Fondo Especial de Desarrollo Universitario (FEDU) de la Universidad Nacional Agraria La Molina y la British Cactus \& Succulent Society (BCSS) a través de la Sociedad Peruana de Cactus y Suculentas (SPECS).

\section{Litertura citada}

Anderson E. 2001. The Cactus Family. Timber Press. Oregon. Inc. U.S.A.

Arakaki M., Ostolaza C., Cáceres F. \& Roque J. 2006. Revista Peruana de Biología. Número especial 13(2): 193s - 219s. El libro rojo de las plantas endémicas del Perú. Ed.: Blanca León et al. Facultad de Ciencias Biológicas UNMSM. Lima - Perú.

Barthlott W. \& Hunt R. 1993. Cactaceae. En: K. Kubitzki (Ed) The families and genera of vascular plants. Vol II: Flowering Plants. Dicotyledons. Springer-Verlag Berlin Heidelberg. Alemania.: 161-196.

Barton A.M. 1986. Spatial variation in the effect of ants on an extrafloral nectary plant. Ecology. 67(2): 495-504.

Brako L. \& Zarucchi J. 1993. Catálogo de las Angiospermas y Gimnospermas del Perú. Missouri Botanical Garden. U.S.A.: 265-309. 
Britton, N. \& Rose J. 1919-1922. The Cactaceae 1-4. Carnegie Institution. U.S.A.

Blom P.E. \& Clark W.H. 1980. Observations of ants (Hymenoptera: formicidae) visiting extrafloral nectaries of the barrel cactus, Ferocactus gracilis Gates (Cactaceae), in Baja California, Mexico. Soutwesternj Naturalist. 25: 181-196.

Bronstein J.L. 1995. The plant-pollinator landscape. In: Hansson L., Fahrig L. and Merriam G. (eds), Mosaic Landscapes and Ecological Processes. Chapman \& Hall, London.

Calderón N. 2003. Estudio taxonómico del género Haageocereus (Familia Cactaceae) en el Departamento de Lima. Tesis para optar el Título de Bióloga. UNALM.

Castro V. 2006. Taxonomía de la familia Cactaceae en el valle de río Chillón, Lima: cerro Umarcata y quebrada Oropel. Tesis para optar el Grado Académico de Magíster Scientiae. UNALM.

Castro V., Eyzaguirre R. \& Ceroni A. 2006. Supervivencia de plántulas de Melocactus peruvianus Vaupel y Haageocereus pseudomelanostele subsp. aurespinus (Rauh \& Backeberg) Ostolaza, en condiciones experimentales. Cerro Umarcata, valle del río Chillón, Lima. Ecología Aplicada. 5 (1 y 2): 61 - 66.

Cerdá X. \& Retana J. 1988. Descripción de la comunidad de hormigas de un prado sabanoide en Canet de Mar (Barcelona). Ecología. 2: 333-341.

Cerdá X., Retana J., Bosch J. \& Alsina A. 1989. Exploitation of food resources by the ant Tapinoma nigerrima (Hym., Formicidae) . Acta Oecologica. 10: 419-429.

Clark R. \& Molina F. 2004. Reproductive ecology of the rare clonal cactus Stenocereus eruca in the Sonora desert. Plant Systematics and Evolution. 247 (3/4): 155164.

Davidson D. \& McKey D. 1993. The evolutionary ecology of symbiotic ant-plant relationships. Journal of Hymenoptera Research. 2(1): 13-83.

Deuth D. 1977. The function of extrafloral nectaires in Aphelandra deppeana Schl. and Cham. (Acanthaceae). Brenesia. 10: 135-145.

De Viana M., Sühring S. \& Manly B. 2000. Application of randomization methods to study the association of Trichocereus pasacana (Cactaceae) With Potential Nurse Plants. Plant Ecology. 156(2): 1-5.

De Viana M., Ortega B., Saravia M. \& Schlumpberger B. 2001. Biología floral y polinizadores de Trichocereus pasacana (Cactaceae) en el Parque Nacional Los Cardones, Argentina. Revista de Biología Tropical. 49(1): 279-285.

Eastwood R. 2006. Congregación de Strymon jacquelinne Nicolay \& Robbins 2005 (Lepidoptera: Lycaenidae): Eumaeini) en nectarios extraflorales del cactus Neoraimondia arequipensis ssp. Gigantean (Werdermann \& Backeberg) Ostolaza. Quepo. 1.3 Ecología. : 28-35.

Farji-Brener A.G. 1993. Influencia de la estacionalidad sobre los ritmos forrajeros de Atta laevigata (Hymenoptera: Formicidae) en una sabana tropical. Revista de Biología Tropical. 41(3): 897-899.

Fleming T. \& Holland J. 1998. The evolution of obligate pollination mutualisms: senita cactus and senita moth. Oecologia. 114: 368-375.
Fleming T., Sahley C., Holland J., Nassar J. \& Hamrick J. 2001. Sonoran desert columnar cacti and the evolution of generalized pollination systems. Ecological Monographs. 71(4): 511-530.

Fonseca C.R. 1999. Amazonian ant-plant interactions and the nesting space limitation hipótesis. Journal of Tropical Ecology. 15: 807-825.

Ford H.A. \& Forde N. 1976. Birds as possible pollinators of Acacia pycnantha. Asutralian Journal of Botany. 24: 793-795.

Fournier L. 1969. Estudio preliminar sobre la floración en el roble de la sabana, Tebebuia pentaphylla (L.) Helmst. Revista de Biología Tropical. 15(2): 259-267.

Gibson A. 1979. Vascular organization in shorts of Cactaceae. 1. Development and morphology of primary vasculature in Pereskioideae and Opuntioideae. American Jornal of Botany. 63: 414 - 426. U.S.A.

Gibson A. \& Nobel P. 1986. The Cactus Primer. Harvard University Press. Cambridge.

Gibson A. \& Rundel P. 2001. Browningia candelaris in the Andean Pre-puna of northern Chile. Cactus \& Succulent Journal (U.S.). 73 (1): 27-33.

Godínez H., Valiente A. \& Rojas A. 2002. The role of seed dispersers in the population dynamics of the columnar cactus Neobuxbaumia tetetzo. Ecology. 83(9): 2617-2629.

Hoffmann A. 1989. Cactáceas. En la flora Silvestre de Chile. Ed. Fundación Claudio Gay.

Holland J. \& Fleming T. 1999. Mutualistic interactions between Upiga virescens (Pyralidae), a pollinating seedconsumer, and Lophocereus schottii (Cactaceae). Ecology. 80(6): 2074-2084.

Holland J. \& Fleming T. 2002. Co-pollinators and specialization in the pollinating seed-consumer mutualism between senita cacti and senita moths. Oecología. 133: 534-540.

Horvitz C.C. \& Schemske D.W. 1984. Effects of nectar feeding ants and ant-tended herbivore on seed production of a neotropical herb. Ecology. 65: 13691378.

Hunt D. 1999. CITES Cactaceae Checklist. 2da edición. Royal Botanic Gardens Kew \& International Organization for Succulent Plant Study. Englang.

Hunt D. 2006. The New Cactus Lexicon. Internacional Cactaceae Systematics Group. dh.

Inouye D.W. \& Taylor O.R. 1979. A temperature region plant-and-seed predator system: consequences of extrafloral nectar secretion by Helianthella quinquenervis. Ecology. 60: 1-7.

IUCN. 2001. IUCN. Red List Categories. Versión 3.1. Gland. Switzerland.

Kearns C., Inouye D. \& Waser N. 1998 Endangered mutualisms: the conservation of plant-pollinator interactions. Annual Review of Ecology and Systematics. 29: 83-112.

Keeler K.H. 1981. Function of Mentzelia nuda (Loasaceae) postfloral nectaries in seed defense. American Journal of Botany. 68: 295-299.

Keopcke H. 1973. Die Lebensformen Band I. Goecke \& Evers- Krefeld.

Koptur S. 1979. Facultative mutualism between weedy verches bearing extrafloral nectaries and weedy ants in California. American Journal of Botany. 66: 1016-1020. 
Krebs C. 2001. Ecology. Benjamin Cumminps, San Francisco.

Leirana J. \& Parra V. 1999. Factors affecting the distribution, abundance and seedling survival of Mammillaria gaumeri, an endemic cactus of coastal Yucatán, México. Journal of Arid Environments. 41: 421-428.

Lerner T., Ceroni A. \& Gonzalez C. 2003. Etnobotánica de la Comunidad Campesina "Santa Catalina de Chongoyape”. Ecología Aplicada. 2(1): 14-20.

Magallanes C. 1997. Cactáceas de la Provincia de Huamanga. Tesis para obtener el Título de Magíster Scientae. UNALM. Lima, Perú.

Márquez C., Vargas H., Snell H., Mauchamp A., Gibas J. \& Tapia W. 2003. ¿Por que tan pocas Opuntias en la Isla Española-Galápagos?. Ecología Aplicada. 2(1): 2129.

Mauseth J. 2000. Theoretical aspects of surface-to-volume ratios and water-storge capacities of succulent shoots. American Journal of Botany. 87: 1107 - 1115.

Mauseth J. \& Kiesling R. 1997. Comparative anatomy of Neoraimondia reoseiflora and Neocardenasia herzogiana (Cactaceae). Haseltonia. 5:37-49.

Mauseth J. \& Plemons B. 1997. Presence of paratracheal water storage tissue does not alter vessel characters in cactus wood. American Journal of Botany. 84: 815 822.

Mauseth J., Kiesling R. \& Ostolaza C. 2002. A Cactus Odissey. Journeys in the wilds of Bolivia, Perú and Argentina. Timber. Oregon. USA.

McAuliffe J. 1984. Prey refugia on the distributions of two sonoran desert cacti. Oecología. 65: 82-85.

McAuliffe J. \& Hendriks P. 1988. Determinants of the vertical distributions of woodpecker nest cavities in the Sahuaro cactus. The Condor. 90: 791-801.

McIntosh M. 2002. Flowering phenology and reproductive output in two sister species of Ferocactus (Cactaceae). Plant Ecology.159: 1-13.

Molina F., Rojas A., Fleming T. \& Valiente A. 2004. Pollination biology of the columnar cactus Pachycereus pecten-aboriginum in north-western México. Journal of Arid Environments. 56: 117-127.

Morris T.I. 1997. Interrelaciones entre olivos, plagas y depredadores. Tesis Doctoral. Universidad de Granada.

Nobel P. 1998. Los Incomparables Agaves y Cactos. Ed. Trillas. México.

Novoa S. 2005. Fenología del cactus Neoraimondia arequipensis subsp. roseiflora (Werdermann \& Backeberg) Ostolaza (Cactaceae) y su relación con la visita de la hormiga Camponotus sp. (Hymenoptera: Formicidae) en el valle del río Chillón, Lima-Perú. Tesis para optar el Título de Biólogo. UNALM.

Novoa S., Castro V., Ceroni A. \& Redolfi I. 2003. Relación entre la hormiga Camponotus sp. (Hymenoptera: Formicidae) y una comunidad de cactus (Cactaceae) en el valle del río Chillón. Ecología Aplicada. 2 (1): 69 73.

Novoa-Shepard S., Redolfi-Pivatto I., Castro-Cepero V. \& Ceroni-Stuva A. 2004. Neoraimondia arequipnesis spp. roseiflora (Werd. \& Backbg) Rauh. (Cactaceae), eje principal de los procesos aéreos en un ecosistema de zona árida. Valle del Río Chillón-Lima-Perú. Resúmenes. IV Congreso Mexicano, III
Latinoamericano y el Caribe de Cactáceas y otras suculentas. Guadalajara, Jalisco, México. : 86.

Novoa S., Ceroni A. \& Arellano C. 2005a. Contribución al conocimiento de la fenología del cactus Neoraimondia arequipensis subsp. roseiflora (Werdermann \& Backeberg) Ostolaza (Cactaceae) en el valle del río Chillón, Lima-Perú. Ecología Aplicada. 4 (1 y 2): 35-40.

Novoa S., Redolfi I. \& Ceroni A. 2005b. Patrón de actividad diario de la hormiga Camponotus sp. en los botones florales del cactus Neoraimondia arequipensis subsp. roseiflora (Werdermann \& Backeberg) Ostolaza. Ecología Aplicada. 4 (1 y 2): 77 - 81.

Novoa S., Redolfi I., Ceroni A. \& Arellano C. 2005c. El forrajeo de la hormiga Camponotus sp. en los botones florales del cactus Neoraimondia arequipensis subsp. roseiflora (Werdermann \& Backeberg) Ostolaza (Cactaceae). Ecología Aplicada. 4 (1 y 2): 83 - 90.

Oficina Nacional de Evaluación de Recursos Naturales (ONERN). 1976. Mapa Ecológico del Perú: Guía Explicativa. Lima-Perú.

Oliveira P.S., Rico-Gray V., Díaz-Castelazo C. \& CastilloGuevara C. 1999. Interaction between ants, extrafloral nectaries and insect herbivores in Neotropical costal sand dunes: herbivore deterrence by visiting ants increases fruit set in Opuntia stricta (Cactaceae). Functional Ecology. 13: 623-631.

Ostolaza C. 1987. La planta del mes. Quepo. Boletín de la sociedad peruana de cactus y suculentas. (1): 11-13.

Ostolaza C. 1996. A closer look at the conservation status of cacto in the vicinity of Lima. Perú. British Cactus and Succulent Journal. U.K. 14(4): 158-174.

Ostolaza C. 2003. Categorización y conservación de Cactáceas peruanas. Zonas Áridas. 7: 199-210.

Ostolaza C., Mitich L. \& King J. 1985. Neoraimondia arequipensis var. roseiflora (Werd. \& Backebg.) Rauh. Cactus and Succulent Journal. U.S. 57: 60-64.

Ostolaza C., Ceroni, A., Dreyfus, G., Calderón N. \& Novoa S. 2003. Cacti of the Huaura river valley, Perú - a conservation survey. British Cactus \& Succulent Journal. 21(2): 87 - 97.

Ostolaza C., Ceroni A., Calderón N. \& Novoa S. 2005. The Huaura valley, Lima, Peru - revisited. British Cactus \& Succulent Journal. U.K. 23(1): 25 - 33.

Ostolaza C., Ceroni A., Calderón N., Alvarez E., Zapata J., Cortez J. \& Salinas L. 2006. Cacti of the Pativilca river basin, Lima, Peru. Cactus World BCSS. U.K. 24(3): 117-128.

Ostolaza C., Ceroni A., Zapata J., Cortez J., Salinas L. \& García E. 2007. Cacti of the Cañete river basin, Lima, Peru. Cactus World BCSS. U.K. 25(4): (en prensa).

Pacheco M., Carrera C., Redolfi I., Ceroni A. \& Castro V. 2004. La comunidad de insectos en los botones abortados de Neoraimondia arequipensis spp. roseiflorae (Wedermann \& Backeberg) Ostolaza (Cactaceae) en el valle del río Chillón. Lima. XLVI Convención Nacional de Entomología. Arequipa. 7 al 11 de Noviembre. Libro de Resúmenes.

Pemberton R.W. 1988. The abundance of plants bearing extrafloral nectaires in Colorado and Mojave desert communities of southern California. Madroño. 35: 238246.

Pickett C.H. \& Clark W.D. 1979. The function of extrafloral nectarines in Opuntia acanthocarpa (Cactaceae). American Journal of Botany. 66: 618-625. 
Redolfi I., Tinaut A., Pascual F. \& Campos M. 2003. Patrón de actividad de Tapinoma nigerrima Nylander y Crematogaster scutellaris Olivier (Hymenoptera: Formicidae) en el cultivo de olivo y en el laboratorio. Zoologica Baetica. 13/14: 37-55.

Rivera L. \& Rodríguez R. 1998. Breeding biology of the crested caracara in the cape region of Baja California, México. Journal of Field Ornithology. 69(2): 160-168.

Rojas A., Valiente A., Arizmendi M., Alcántara A. \& Arita H. 1999. Seasonal distribution of the long-nosed bat (Leptonicteris curosoae) in the North America: does a generalized migration pattern really exist?. Journal of Biogeography. 26: 1065-1077.

Roque J. \& Ramírez E.K. 2005. Palos de lluvia y cactáceas. Quepo. Boletín de la sociedad peruana de cactus y suculentas. (19): 84-91.

Ruffener G.A. \& Clark W.D. 1986. Extrafloral nectar of Ferocactus acanthodes (Cactaceae): composition and its importance to ants. American Journal of Botany. 73: 185-189.

Sarmiento G. 1984. Los ecosistemas y la ecósfera. Editorial Blume. Barcelona.

Sahley C. 1996. Bat and huming bird pollination of an autotetraploid columnar cactus, Weberbauerocereus webwerbaueri (Cactaceae). American Journal of Botany. 83(10): 1329-1336.

Schemske D.W. 1980. The evolutionary significance of extrafloral nectar production by Costus woodsonii (Zingiberaceae): an experimental analysis of ant protection. Journal of Ecology. 68: 663-669.

Scobell S. \& Scout P. 2002. Visitor and floral traits of huming birds-adapted cactus (Echinocereus coccineus) show only minor variation along an elevational gradient. The American Midland Naturalist. 17(1): 1-15.

Stephenson A.G. 1982. The role of extrafloral nectaries of Catalpa speciosa in limiting herbivory and increasing fruit production. Ecology. 63: 663-669.

Teixeira V. 2006. Análisis de la diversidad de la composición de Cactáceas y su relación con los factores edáficos en el valle del río Chillón, Lima: cerro Umarcata y quebrada Orobel. Tesis para optar el Grado Académico de Magíster Scientiae. UNALM.

Teixeira V., Castro V., Ceroni A. \& Eyzaguirre R. 2004. Diversidad y densidad de la comunidad de cactáceas en el valle del río Chillón: cerro Umarcata y quebrada Orobel y su relación con los factores edáficos. Ecología Aplicada. 3 (1 y 2): 1 - 8.

Terrazas S. \& Mauseth J. 2002. Cacti. Biology and uses. Chapter 2: Shoot Anatomy and Morphology. Edited by Park S. Nobel. University of California Press. U.S.A.

Turner R., Alcorn S., Olin G. \& Booth J. 1966. The influence of shade, soil, and water on Saguaro seedling establishment. Botanical Gazette. 127(2-3): 95-102.

Valiente A. \& Ezcurra E. 1991. Shade as a cause of the association between the cactus Neobuxbaumia tetetzo and the nurse plant Mimosa luisana in the Tehuacán Valley, México. Journal of Ecology. 79: 961-971. U.S.A.

Valiente A., Bolongaro A., Briones O., Ezcurra E., Rosas M., Núñez H., Barnard G. \& Vasquez E. 1991 a. Spatial relationships between cacti and nurse shrubs in a semiarid environment in central México. Journal of Vegetation Science. 2: 15-20. Sweden.

Valiente A., Vite F. \& Zavala A. 1991 b. Interaction between the cactus Neobuxbaumia tetetzo and the nurse shrub Mimosa luisana. Journal of Vegetation Science. 2:11-14. Sweden.

Valiente A., Del Coro M., Rojas A. \& Dominguez L. 1996. Ecological relationships between columnar cacti and nectar-feeding bats in México. Journal of Tropical. Ecology. 12(1): 103-119.

Valiente A., Rojas A., Del Coro M. \& Davila P. 1997. Pollination Biology of two columnar cacti (Neubuxbaumia mezcalaensis and Neobuxbaumia macrocephala) in the Tehuacan Valley, central Mexico. American Journal of Botany. 84(4): 452-455.

Wäckers F.L \& Bonifay C. 2004. How to be sweet?. Extrafloral nectar allocation by Gossypium hirsutum fits optimal defense theory predictions. Ecology. 85(6): 1512-1518.

Wagner D. 1997. The influence of ant nests on Acacia seed production, herbivory and soil nutrients. Journal of Ecology. 85: 83-93.

Weberbauer A. 1945. El mundo vegetal de los Andes Peruanos: Estudio Fitogeográfico. $2^{\circ}$ Edición. Estación Experimental Agrícola La Molina. Perú.

\footnotetext{
1Jardín Botánico “Octavio Velarde Núñez”. aceroni@lamolina.edu.pe, vcastro@lamolina.edu.pe

${ }^{2}$ Laboratorio de Control Biológico y Ecología de Artrópodos, rhi@lamolina.edu.pe. Departamento Académico de Biología. Facultad de Ciencias. Universidad Nacional Agraria La Molina. Av. La Molina s/n, Apartado Postal 12056, Lima100. Perú, 3: vteixeira@pucp.edu.pe, Pontificia Universidad Católica del Perú (PUCP), Apartado 1761.
} 